\title{
Quantum well saturable absorber mirror with electrical control of modulation depth
}

\author{
Liu, Xiaomin; Rafailov, Edik U.; Livshits, Daniil; Turchinovich, Dmitry
}

Published in:

Applied Physics Letters

Link to article, DOI:

10.1063/1.3474799

Publication date:

2010

Document Version

Publisher's PDF, also known as Version of record

Link back to DTU Orbit

Citation (APA):

Liu, X., Rafailov, E. U., Livshits, D., \& Turchinovich, D. (2010). Quantum well saturable absorber mirror with electrical control of modulation depth. Applied Physics Letters, 97, 1-3. https://doi.org/10.1063/1.3474799

\section{General rights}

Copyright and moral rights for the publications made accessible in the public portal are retained by the authors and/or other copyright owners and it is a condition of accessing publications that users recognise and abide by the legal requirements associated with these rights.

- Users may download and print one copy of any publication from the public portal for the purpose of private study or research.

- You may not further distribute the material or use it for any profit-making activity or commercial gain

- You may freely distribute the URL identifying the publication in the public portal

If you believe that this document breaches copyright please contact us providing details, and we will remove access to the work immediately and investigate your claim 


\title{
Quantum well saturable absorber mirror with electrical control of modulation depth
}

\author{
Xiaomin Liu, ${ }^{1}$ Edik U. Rafailov, ${ }^{2}$ Daniil Livshits, ${ }^{3}$ and Dmitry Turchinovich ${ }^{1, a)}$ \\ ${ }^{1}$ DTU Fotonik-Department of Photonics Engineering, Technical University of Denmark, DK-2800 Kgs. \\ Lyngby, Denmark \\ ${ }^{2}$ School of Engineering, Physics, and Mathematics, University of Dundee, Dundee DD1 4HN, United \\ Kingdom \\ ${ }^{3}$ Innolume GmbH, Konrad-Adenauer-Allee 11, 44263 Dortmund, Germany
}

(Received 17 June 2010; accepted 9 July 2010; published online 2 August 2010)

\begin{abstract}
We demonstrate a quantum well (QW) semiconductor saturable absorber mirror (SESAM) comprising low-temperature grown InGaAs/GaAs QWs incorporated into a $p$ - $i-n$ structure. By applying the reverse bias voltage in the range $0-2 \mathrm{~V}$ to the $p-i-n$ structure, we were able to change the SESAM modulation depth in the range $2.5-0.5 \%$, as measured by nonlinear reflectivity of 450 fs long laser pulses with $1065 \mathrm{~nm}$ central wavelength, in the pump fluence range $1.6-26.7 \mu \mathrm{J} / \mathrm{cm}^{2}$. This electrical control of the modulation depth is achieved by controlling the small-signal loss of the SESAM via quantum-confined Stark effect in the QWs. () 2010 American Institute of Physics. [doi:10.1063/1.3474799]
\end{abstract}

Semiconductor saturable absorber mirrors (SESAMs) are used for mode-locking and control of ultrafast lasers (see, e.g., Ref. 1 and references therein). The modulation depththe value of saturable loss of the SESAM, is an important parameter governing the self-starting and stability of laser mode-locking. In this letter, we demonstrate an electrically controlled SESAM (E-SESAM) for the wavelength around $1065 \mathrm{~nm}$, based on the low-temperature (LT) grown InGaAs/ GaAs quantum wells (QWs) embedded into a $p-i-n$ structure. By applying the external bias on the $p-i-n$ structure, the E-SESAM nonlinear reflectivity could be modified strongly.

The SESAM action is based on the saturation of the optical loss in the absorber section at higher optical intensity, thus resulting in a higher SESAM reflectivity for stronger optical signals. The efficient mode-locking of an ultrafast laser is additionally dependent on the efficient ultrafast recovery of the saturable loss in the SESAM. This is ensured by the quick depletion of the photoexcited electronic states in the saturable absorber, which can be achieved by, e.g., capture of the photoexcited carriers onto lattice defects, ${ }^{2,3}$ and/or carrier sweep-out in an applied electric field. ${ }^{4}$ The dynamical screening effect ${ }^{5,6}$ could also have an impact on the ultrafast dynamics in biased QWs or quantum dots (QDs).

Laser mode-locking and control with E-SESAMs based on $\mathrm{QWs}^{7,8}$ and QDs ${ }^{9,10}$ was recently demonstrated, by applying an electric field on the saturable absorber section. For example, in Ref. 8, the mode-locked laser stability could be manipulated, and pulse shortening from 50 ps down to $20 \mathrm{ps}$ was observed, accompanied by the spectral expansion of the laser signal from 0.3 to $0.8 \mathrm{~nm}$, which was attributed to a speed-up of the SESAM recovery rate due to the enhanced carrier sweep-out at higher bias fields. In Ref. 10, a pulse shortening from 17.4 to $6.4 \mathrm{ps}$, and a spectral shift from 1280 to $1282 \mathrm{~nm}$ were observed, with applying the reverse bias to the E-SESAM.

However, the laser is a complex system, where the SESAM action is in interplay with gain dynamics, gain cur-

${ }^{a)}$ Electronic mail: dmtu@fotonik.dtu.dk. vature, and other wavelength-, power-, and time-dependent loss factors in the oscillating laser cavity. In this work, we performed a direct experiment to characterize the E-SESAM action alone, by measuring the nonlinear reflectivity of femtosecond laser pulses as a function of both pump fluence and the reverse bias on the E-SESAM.

Our $p$ - $i$ - $n$-junction-based E-SESAM structure was grown on an n-doped GaAs substrate using a standard molecular beam epitaxy. It had an n-doped 34-period long GaAs $/ \mathrm{Al}_{0.9} \mathrm{Ga}_{0.1}$ As Bragg reflector with the stop band covering the range 1014-1126 nm, followed by an undoped saturable absorber (QWs) section, and a $p$-doped GaAs cap layer, as shown in Fig. 1(a). The saturable absorber section
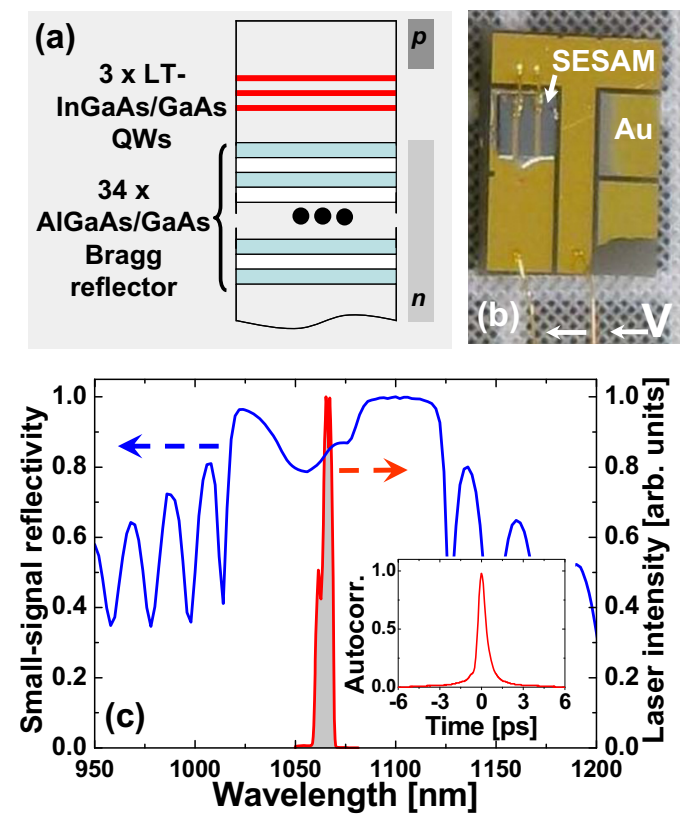

FIG. 1. (Color online) (a) Schematic of an E-SESAM. (b) Photograph of an E-SESAM chip; Au: reference golden mirror and V: external bias contacts. (c) E-SESAM small-signal reflectivity spectrum at zero external bias, and the intensity spectrum of the femtosecond laser output used in the nonlinear reflectivity experiment. Inset: laser pulse autocorrelation. 
featured 3 LT- $\operatorname{In}_{0.2} \mathrm{Ga}_{0.8} \mathrm{As} / \mathrm{GaAs}$ QWs with the QW width of $8 \mathrm{~nm}$, additionally surrounded by $\mathrm{GaAs}_{0.75} \mathrm{P}_{0.25}$ barriers on each side for strain compensation in the QWs. The total thickness of the intrinsic section was $155 \mathrm{~nm}$, and the potential drop of the $p-i-n$ junction was approximately $1.0 \mathrm{~V}$, resulting in the built-in electric field on the QWs of around 65 $\mathrm{kV} / \mathrm{cm}$. The flat electrode was applied to the back of the n-doped GaAs substrate, and narrow stripe electrodes separated by approximately $300 \mu \mathrm{m}$ were deposited on the $p$-doped cap layer, providing the free optical access to the SESAM. In Figs. 1(b) the photograph of the E-SESAM chip is shown. The E-SESAM area on the chip was about 1 $\times 1 \mathrm{~mm}^{2}$.

In Fig. 1(c) we show a small-signal reflectivity spectrum of the E-SESAM at zero external bias, and the intensity spectrum of the output of the femtosecond laser used in the nonlinear reflectivity measurements. The peak of the optical absorption of the QWs in the E-SESAM at zero external bias is around $1056 \mathrm{~nm}$. The laser was a home-built monolithic $\mathrm{Yb}$ fiber femtosecond laser ${ }^{11}$ operating at central wavelength of $1065 \mathrm{~nm}$ at the repetition rate of $45 \mathrm{MHz}$, and providing the pulses of approximately 450 fs duration (full width at half maximum), as assumed from the $650 \mathrm{fs}$ long autocorrelation [see inset of Fig. 1(c)]. The laser output was focused onto the surface of the E-SESAM in between the stripe electrodes, and the incident and reflected laser power was measured using the calibrated powermeters. The laser pump fluence on the E-SESAM was varied in the range $1.6-26.7 \mu \mathrm{J} / \mathrm{cm}^{2}$ with a $\lambda / 2$-plate and a polarizer. A reference golden mirror with the reflectivity of $98 \%,{ }^{12}$ also deposited on the E-SESAM chip, was used for absolute calibration of the reflectivity values.

In Fig. 2(a) the dependency of the E-SESAM reflectivity $R$ on the pump fluence $F_{p}$, for different values of applied reverse bias $V$ in the range $0-2 \mathrm{~V}^{13}$ is shown. For all values of $V$ we observed a typical SESAM behavior, as reflectivity grows with increase in the pump fluence in the whole range of the $F_{p}$. We note here, that within the pump fluence range available to us $\left(1.6-26.7 \mu \mathrm{J} / \mathrm{cm}^{2}\right)$ we could not reach the full saturation of QW absorption. With increase in $V$, the overall E-SESAM reflectivity increases, thus showing a reduction in E-SESAM loss, with a significant decrease in the slope of the dependency $R\left(F_{p}\right)$. The dynamics of $R(V)$ is most dramatic at weaker pump fluence, as compared to the stronger pump. In Fig. 2(b), the dependency $R(V)$ is shown for two extreme values of pumping fluences available in this experiment. At weakest pump, the $R(V)$ first grows with the reverse bias in the range $0-1 \mathrm{~V}$ [corresponding to the total electric field on the QWs $\left.E_{\text {tot }}=65-130 \mathrm{kV} / \mathrm{cm}\right]$, and then shows an oscillation between 1 and $2 \mathrm{~V}\left[E_{\mathrm{tot}}=194 \mathrm{kV} / \mathrm{cm}\right]$, with the total voltage-induced reflectivity modulation of about 3\%. At strongest pump $R(V)$ also grows in the range $V=0-1 \mathrm{~V}$, and then rolls-off at higher values of $V$, but with the total modulation of only about 1\%. In Fig. 2(c) the modulation depth $\Delta R$ achieved in this experiment - the difference between the $R$ values measured at the extreme pump fluences [Fig. 2(b)] is shown as a function of $V$. The dependency $\Delta R(V)$ is clearly dominated by the dependency of $R(V)$ at weakest pump. $\Delta R$ is the largest at reverse bias $V=0$ and reaches about $2.5 \%$. At $V=1 \mathrm{~V}$ it reaches a local minimum of about $1 \%$, and then shows an oscillation before reaching its absolute minimum of around $0.5 \%$ at $V=2 \mathrm{~V}$.
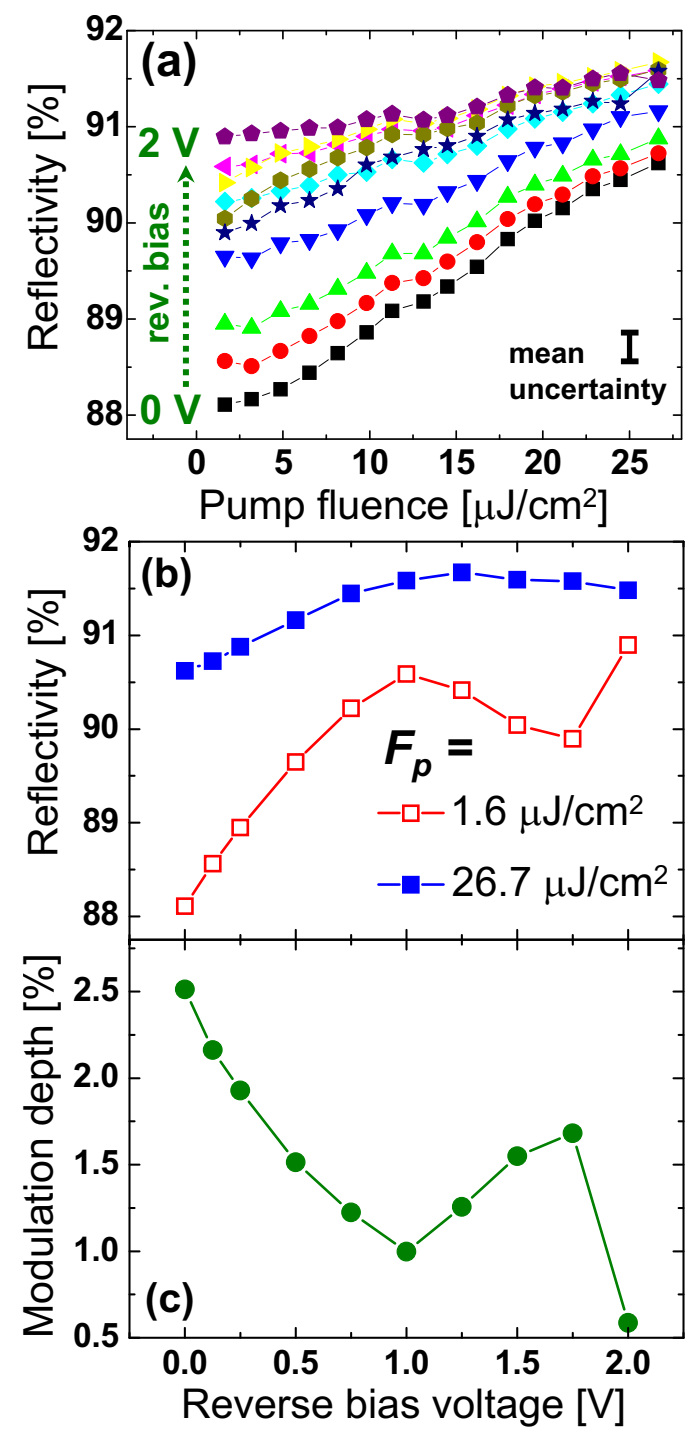

FIG. 2. (Color online) (a) Reflectivity of E-SESAM as a function of pump fluence, at different values of reverse bias in the range 0-2 V. (b) Reflectivity of the E-SESAM at pump fluence of 1.6 and $26.7 \mu \mathrm{J} / \mathrm{cm}^{2}$, as a function of reverse bias. (c) Modulation depth of the E-SESAM as a function of reverse bias.

We also measured the spectrum and the autocorrelation of the reflected laser signal as a function of both $F_{p}$ and $V$, but neither the reflected laser pulse shortening ${ }^{14}$ nor its spectral expansion was observed within the accuracy of our measurements. We note that in Ref. 4 the recovery time of a QD saturable absorber with applied electric field was found to decrease from 62 ps to 700 fs by enhanced carrier sweep-out, which is still longer than 450 fs pulses, used in our experiments.

The application of the transverse electric field to the QW results in a well-known quantum-confined Stark effect (QCSE). ${ }^{15}$ The QCSE manifests itself in the reduction in the optical transition energy (the Stark shift) as a result of the band structure tilt; and in the reduction in the optical absorption coefficient due to the weaker overlap of the spatially separated electron and hole wave functions. The spectrum of our laser is situated at the longer wavelength than the peak of the QW absorption (i.e., the strongest dip in the E-SESAM reflectivity). The application of the reverse bias to the E-SESAM, and therefore the enhancement of the QCSE already present due to the built-in $p-i-n$ field of $65 \mathrm{kV} / \mathrm{cm}$, 

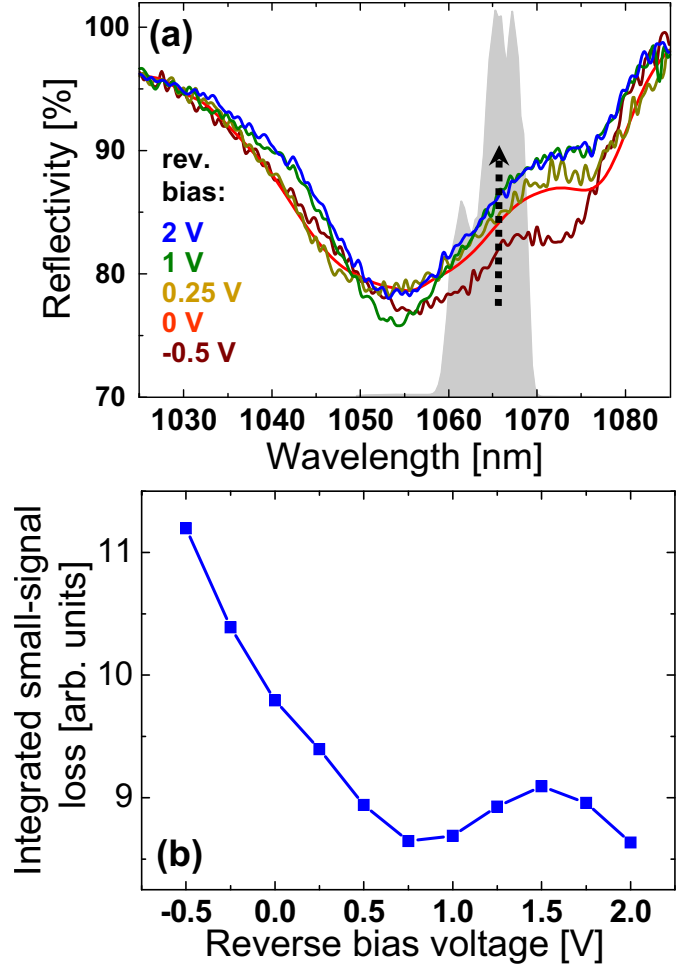

FIG. 3. (Color online) (a) Small-signal reflectivity of the E-SESAM measured at selected bias values. Laser spectrum is shown on the background. (b) Calculated small-signal loss of E-SESAM as experienced by the laser output, as a function of reverse bias.

could thus lead to two following conflicting consequences: (i) Stark-shifting the QW absorption maximum into the laser spectrum, which is positioned at longer wavelength, thus increasing the E-SESAM loss at the laser wavelength and (ii) the overall reduction in the optical absorption coefficient of the QWs. In Fig. 3(a) small-signal reflectivity spectra of the E-SESAM in the vicinity of the laser spectrum, taken at different bias voltages, are presented, showing a rather complex modification. One can see that both manifestations of the QCSE are present in these spectra but their respective contributions are different in different spectral ranges. The Stark shift mostly dominates around the wavelength of $1045 \mathrm{~nm}$, whereas the $Q W$ absorption change with applied bias seems to be the dominating effect around the central wavelength of the laser at $1065 \mathrm{~nm}$. Here, in addition to the reverse bias in the range $0-2 \mathrm{~V}$, we also used a small forward bias up to 0.5 $\mathrm{V}^{13}\left[E_{\mathrm{tot}}=32 \mathrm{kV} / \mathrm{cm}\right]$ to see the effect of QCSE reduction. It is clear that the QCSE from the $p-i-n$ built-in electric field alone already has a significant impact on the optical properties of the SESAM.

We quantified the integrated small-signal loss, experienced by the laser in the E-SESAM at a given value of bias voltage by calculating the overlap integral between the laser intensity spectrum $I(\lambda)$ and the SESAM loss for different values of applied bias; $S(V)=\int_{\lambda} I(\lambda)[1-R(V, \lambda)] d \lambda$. Here $R(V, \lambda)$ is the SESAM small-signal reflectivity spectrum at specific value of bias voltage. The dependency $S(V)$ is shown in Fig. 3(b). It is nearly identical to the bias voltage dependency of the modulation depth $\Delta R(V)$, which is in turn dominated by the weak-pump reflectivity dependency on $V$ [see Figs. 2(b) and 2(c)].
This demonstrates that the achieved control of the modulation depth of the E-SESAM is mostly due to the modification of its small-signal loss at the laser wavelength by the QCSE. The observed decrease in the slope of $R\left(F_{p}\right)$ with increase in $V$ is thus a natural consequence of this effect. As the QWs become more transparent for the pump laser, $R\left(F_{p}\right)$ will depend less on the saturable loss, and in the limit of zero QW absorption will approach the reflectivity of a Bragg reflector (neglecting the nonsaturable loss).

In conclusion, we have demonstrated a QW-based E-SESAM for the wavelength of $1065 \mathrm{~nm}$. Its modulation depth in the pump fluence range $1.6-26.7 \mu \mathrm{J} / \mathrm{cm}^{2}$ can be tuned in the range $2.5-0.5 \%$, i.e., by a factor of 5 , by application of the external reverse bias in the range $0-2 \mathrm{~V}$, which also has a strong effect on the slope of the dependency $R\left(F_{p}\right)$, important for the self-starting of laser mode-locking. The electrical control of the E-SESAM is based on the QCSE, which in this case was found to lead predominantly to the decrease in the QW small-signal absorption at the pump laser wavelength. The E-SESAMs can be used for active stabilization of ultrafast lasers and complement the passive nonlinear-optical control methods. ${ }^{11,16,17}$ We note that the reshaping of E-SESAM reflectivity spectrum by QCSE could be the reason for the laser spectral shift, observed in Ref. 10.

We are grateful to Danish Advanced Technology Foundation (HTF) and EU FP7 Programme (FAST-DOT, Grant No. 224338) for financial support; and to K. Yvind, A. Isomäki, and J. M. Hvam (DTU Fotonik) for valuable discussions.

${ }^{1}$ U. Keller, Nature (London) 424, 831 (2003)

${ }^{2}$ L. R. Brovelli, U. Keller, and T. H. Chiu, J. Opt. Soc. Am. B 12, 311 (1995).

${ }^{3}$ A. Jasik, J. Muszalski, K. Pierscinski, M. Bugajski, V. G. Talalaev, and M. Kosmala, J. Appl. Phys. 106, 053101 (2009).

${ }^{4}$ D. B. Malins, A. Gomez-Iglesias, S. J. White, W. Sibbett, A. Miller, and E. U. Rafailov, Appl. Phys. Lett. 89, 171111 (2006).

${ }^{5}$ D. Turchinovich, P. U. Jepsen, B. S. Monozon, M. Koch, S. Lahmann, U. Rossow, and A. Hangleiter, Phys. Rev. B 68, 241307(R) (2003).

${ }^{6}$ D. Turchinovich, B. S. Monozon, and P. U. Jepsen, J. Appl. Phys. 99, 013510 (2006).

${ }^{7}$ B. Stormont, E. U. Rafailov, I. G. Cormack, A. Mooradian, and W. Sibbett, Electron. Lett. 40, 732 (2004).

${ }^{8}$ A. Isomäki, A. Vainionpää, S. Suomalainen, and O. G. Okhotnikov, Proc. SPIE 5958, 59580R (2005).

${ }^{9}$ A. A. Lagatsky, E. U. Rafailov, W. Sibbett, D. A. Livshits, A. E. Zhukov, and V. M. Ustinov, IEEE Photonics Technol. Lett. 17, 294 (2005).

${ }^{10}$ S. A. Zolotovskaya, K. G. Wilcox, A. Abdolvand, D. A. Livshits, and E. U. Rafailov, IEEE Photonics Technol. Lett. 21, 1124 (2009).

${ }^{11}$ D. Turchinovich, X. Liu, and J. Lægsgaard, Opt. Express 16, 14004 (2008).

${ }^{12}$ M. A. Ordal, L. L. Long, R. J. Bell, S. E. Bell, R. R. Bell, R. W. Alexander, Jr., and C. A. Ward, Appl. Opt. 22, 1099 (1983).

${ }^{13}$ In order to prevent the electric breakdown of the $p-i$ - $n$ structure, we limited the reverse bias to $2 \mathrm{~V}$ in all measurements, and the forward bias to $0.5 \mathrm{~V}$ in $R(V, \lambda)$ measurements

${ }^{14}$ J.-C. Wang, C.-K. Sun, and J.-K. Wang, Appl. Phys. Lett. 89, 231106 (2006).

${ }^{15}$ D. A. B. Miller, D. S. Chemla, T. C. Damen, A. C. Gossard, W. Wiegmann, T. H. Wood, and C. A. Burrus, Phys. Rev. Lett. 53, 2173 (1984).

${ }^{16}$ X. Liu, J. Lægsgaard, and D. Turchinovich, Opt. Lett. 35, 913 (2010).

${ }^{17}$ X. Liu, J. Lægsgaard, and D. Turchinovich, Opt. Express 18, 15475 (2010). 